\title{
Abordagem cognitiva na intervenção terapêutica ocupacional com indivíduos com Doença de Alzheimer
}

\section{Cognitive approach in occupational therapeuticintervention with individuals affected by Alzheimer's disease}

Resumo

A doença de Alzheimer compromete progressivamente, em razão da neurodegeneração, a integração cognitiva e os componentes cognitivos de desempenho, levando ao comprometimento das áreas de desempenho (atividades de vida diária, atividades de trabalho e produtivas, e atividades de lazer ou diversão) nos contextos em que o indivíduo está inserido, gerando déficit no desempenho ocupacional. Assim, o objetivo deste trabalho é evidenciar, através da revisão bibliográfica, a importância da intervenção terapêutica ocupacional com indivíduos com doença de Alzheimer, utilizando uma abordagem cognitiva, que vise à manutenção das habilidades cognitivas do indivíduo com doença de Alzheimer para um desempenho ocupacional competente pelo maior período de tempo. Observa-se a relação direta entre processo de envelhecimento, doença de Alzheimer e desempenho ocupacional. Nesse contexto, o terapeuta ocupacional é inserido no tratamento do indivíduo com doença de Alzheimer, pois tem meios para abordar os componentes afetados pela doença, através de um processo terapêutico estruturado. Ao final, conclui-se que as intervenções cognitivas no estágio inicial são utilizadas para organizar as atividades do cotidiano do indivíduo com doença de Alzheimer e, consequentemente, promover melhor qualidade de vida.

Suzana Elisa Sedrez Corrêa" Derivan Brito da Silva*

Palavras-chave:

Terapia

Ocupacional.

Doença de

Alzheimer.

Cognição. Idoso.

\footnotetext{
Universidade Federal do Paraná, Curso de Terapia Ocupacional. Coordenação. Rua Pe. Camargo, 280, $3^{\circ}$ and, Alto da Glória 80060-240 - Curitiba, PR, Brasil

Correspondência / Correspondence

Suzana Elisa Sedrez Corrêa

E-mail: suzanaelisa_to@hotmail.com
} 


\section{Abstract}

Due to neurological degeneration, Alzheimer's disease gradually affects the cognitive abilities. Subsequently, this compromises the occupational performance in all areas: self-care, productivity and leisure. This paper presents the results of a literature review with respect to the importance of occupational therapy interventions using cognitive approach with individuals with Alzheimer's disease, concerninfg maintenance of cognitive abilities and occupational performance of individuals affected by Alzheimer's

Key words: Occupational Therapy. Alzheimer Disease. Cognition. Aged. disease. It underlines the direct relationship between the process of aging, Alzheimer's disease and occupational performance. Within this context, the role of the occupational therapist includes the use of various approaches to address the affected occupational performance components. The authors conclude that a cognitive approach can effectively be used to organise the activities of daily living of individuals in the early stages of Alzheimer's Disease and to promote a better quality of life.

\section{INTRODUÇ̃̃O}

O indivíduo idoso vivencia transformações biológicas, físicas, psicológicas e sociais ocasionadas pelo processo gradual, irreversível, progressivo, inevitável e universal de envelhecimento, definido como envelhecimento primário. Somado a isto, o indivíduo com doença de Alzheimer passa por um processo patológico de envelhecimento, definido como envelhecimento secundário, no qual ocorre degeneração do sistema nervoso central, resultando na perda progressiva das habilidades cognitivas. ${ }^{1,2}$

Por ser a perda cognitiva um dos principais fatores relacionado à redução do desempenho ocupacional do indivíduo com doença de Alzheimer, o objetivo deste artigo é apresentar a intervenção terapêutica ocupacional com indivíduos com doença de Alzheimer, com enfoque na manutenção das habilidades cognitivas no estágio inicial da doença. Para tanto, apresenta-se os aspectos da velhice e da doença de Alzheimer, a fundamentação terapêutica ocupacional para a intervenção proposta e a intervenção propriamente dita.

\section{MATERIAL E MÉTODO}

Para levantamento de estudos publicados sobre o tema da pesquisa, adotou-se como critério de inclusão a revisão bibliográfica de livros, artigos de revistas e artigos em base de dados, publicados no período de 1998 a 2006. Foram consultadas as bases de dados Bireme, SciELO e OTseeker. Do resultado do levantamento, foram selecionados e agregados os dados e informações sobre os comprometimentos cognitivos manifestados nos indivíduos portadores de doença de Alzheimer e das intervenções cognitivas, como 
forma de manter as habilidades cognitivas desses indivíduos. Outras formas de intervenção e pesquisas anteriores a 1998 foram excluídas da pesquisa.

\section{VELHICE E DOENÇA DE ALZHEIMER}

Segundo a Organização Mundial da Saúde, ${ }^{3}$ a definição de idoso inicia aos 65 anos de idade nos países desenvolvidos, e aos 60 anos nos países em desenvolvimento. No Brasil, de acordo com o Estatuto do Idoso, as pessoas com idade igual ou superior a 60 anos são reconhecidas como idosas. ${ }^{4,5,6}$

No Brasil, houve um crescimento expressivo da expectativa de vida, levando ao aumento do número de idosos. ${ }^{4}$ Mas este crescimento não vem sendo acompanhado por uma melhor qualidade de vida, principalmente em decorrência da alta incidência de doenças, destacando-se as de natureza crônica, como a doença de Alzheimer. ${ }^{7}$ Esta, apesar de se manifestar a partir dos 50 anos de idade, aumenta significativamente sua incidência ao longo dos anos, caracterizando-se, portanto, como uma doença da velhice. ${ }^{1}$

$\mathrm{Na}$ estimativa do Ministério da Saúde, de 600 mil a 1 millhão dos brasileiros apresentam a doença de Alzheimer. Em todo mundo, 15 milhões de pessoas têm a doença de Alzheimer. ${ }^{8}$ Com o aumento da expectativa de vida, estima-se que em 2040 o número de indivíduos com demência, não somente Alzheimer, será de 81,1 milhões. ${ }^{9}$
A doença de Alzheimer é uma doença neurológica degenerativa, lenta e progressiva. ${ }^{5} \mathrm{O}$ indivíduo atingido por ela apresenta progressiva perturbação de múltiplas funções cognitivas, incluindo memória, atenção e aprendizado, pensamento, orientação, compreensão, cálculo, linguagem e julgamento. O comprometimento das funções cognitivas é comumente acompanhado, e ocasionalmente precedido, por deterioração do controle emocional, comportamento social ou motivação. ${ }^{8}$ Estes comprometimentos levam à alteração do desempenho ocupacional do indivíduo com doença de Alzheimer. ${ }^{10}$ Assim, para compreensão do impacto da doença de Alzheimer na funcionalidade, faz-se necessário abordar o processo do envelhecimento cognitivo.

A teoria de mecanismos de ganhos e perdas é uma das teorias que embasam a compreensão do processo de envelhecimento cognitivo. Esta teoria se baseia no fato de que perdas ocorrem desde o nascimento e que, no percurso da vida, inclusive na velhice, estabelece-se uma proporção entre perdas, ganhos e funções mantidas. ${ }^{11}$

Há complexa interação das diferentes funções cognitivas, sendo que na velhice consideram-se ganhos uma função cognitiva que: melhora com a idade; mantém-se, apesar do envelhecimento; tem um pequeno declínio se comparada com outra; forma-se a partir de novas estratégias cognitivas para suprir dificuldades em outras funções. Além disso, a teoria de mecanismos de ganhos e perdas considera também as 
perdas, acreditando haver uma proporção estabelecida entre perdas, ganhos e funções mantidas, sendo considerada uma teoria otimista comparando-se às teorias que focalizam apenas perdas. Conclui-se que a teoria de mecanismos de ganhos e perdas é mais realista e consegue abordar a complexidade do envelhecimento cognitivo. ${ }^{11}$

Deriva desta teoria a abordagem de ganhos em funcão de perdas. Esta abordagem é bastante conhecida em terapias com indivíduos com lesões cerebrais. Baseia-se em ganhos para suprir as dificuldades que surgem gradativamente nos processos demenciais, focalizando ganhos específicos. Esses ganhos se referem a uma nova aquisição ou a uma diferente forma de desenvolver determinada tarefa. Pode ocorrer através de níveis orgânicos, com o acontecimento do fenômeno da plasticidade, no qual áreas cerebrais assumem funções de outras áreas lesadas ou ineficazes; ou em nível cognitivo, que envolve a utilização de diferentes estratégias, como estratégias de compensação, desenvolvimento da expertise e a formação de ambientes colaborativos. ${ }^{11}$

Esclarece-se que: ${ }^{11}$

- as estratégias de compensação são novas técnicas utilizadas para realizar tarefas antigas;

- o desenvolvimento da expertise é a experiência e o conhecimento avançado que o indivíduo adquire ao longo dos anos para realizar determinada ativida- de, servindo como facilitadora para que o indivíduo realize uma atividade;

- a formação de ambientes colaborativos é a utilização de estratégias que facilitem a linguagem e comunicação do indivíduo em seu meio, por exemplo, dicas e interações dialógicas.

A abordagem de ganhos em função de perdas, derivada da teoria de mecanismos de ganhos e perdas, parece ser a mais promissora, pois acredita na capacidade do idoso para elaborar novas estratégias adaptativas às suas dificuldades e permite elaborar um programa de intervenção para o declínio cognitivo patológico. ${ }^{11}$

A partir das elucidações acerca do indivíduo com doença de Alzheimer e os aspectos a ele inerentes, será apresentada a fundamentação para a intervenção proposta neste artigo.

\section{FUNDAMENTAÇÃO TEÓRICA PARA A INTERVENCẼOO DO TERAPEUTA OCUPACIONAL COM INDIVÍDUOS COM DOENÇA DE ALZHEIMER}

A intervenção terapêutica ocupacional, em seus contextos de saúde e sociedade, pressupõe um saber teórico-prático, organizando um modo específico de intervenção mediante o qual confirma ou contesta este saber. ${ }^{12}$

A terapia ocupacional mostra-se inerente à ocupação humana. A busca pelo signi- 
ficado da ocupação humana direcionou os primeiros princípios teóricos da profissão, ${ }^{13}$ que foram descritos em três considerações: ${ }^{9}$

- a primeira é que os humanos foram conhecidos como possuidores de uma natureza ocupacional;

- a segunda, que a doença foi vista como sendo um potencial para interromper ou romper a ocupação;

- a última, que a ocupação foi reconhecida como um organizador natural do comportamento humano, que poderia ser usada terapeuticamente para refazer ou reorganizar o comportamento cotidiano.

De acordo com esses pressupostos, identifica-se que o indivíduo com doença de Alzheimer tem sua essência ocupacional rompida pelos sintomas trazidos por esta, podendo, no seu estágio inicial, ser utilizada a ocupação para reorganizar o comportamento cotidiano.

Esta ocupação, inerente à pessoa e ao ambiente no qual está inserida, caracteriza o desempenho ocupacional. Nessa perspectiva, esclarece-se que os elementos do desempenho ocupacional são as áreas, os componentes e os contextos de desempenho. ${ }^{14,15}$

As áreas de desempenho são "amplas categorias de atividade humana, que fazem parte, tipicamente, da vida cotidiana", subdivida em: atividades de vida diária, atividades de trabalho e produtivas, e atividades de lazer ou de diversão.
Os componentes de desempenho são compreendidos como "capacidades humanas fundamentais que - em graus variados e em diferentes combinações - são necessárias para o engajamento bem-sucedido nas áreas de desempenho". Estes componentes estão divididos em sensório-motor, integração cognitiva e componentes cognitivos, habilidades psicossociais e componentes psicológicos.

Os contextos de desempenho caraterizam-se como "situações ou fatores que influenciam o engajamento da pessoa nas áreas de desempenho desejadas e/ou necessárias", consistindo estes em aspectos temporais e ambiente.

A adequada relação entre áreas, contextos e componentes de desempenho determinam o sucesso do desempenho ocupacional, sendo este sucesso a preocupação final da intervenção terapêutica ocupacional. ${ }^{16}$ Este sucesso é caracterizado como "performance ocupacional competente", a qual depende da interação complexa entre o indivíduo, o ambiente e as coisas que ele faz. ${ }^{17}$ Acrescenta-se que um equilíbrio adequado no desempenho ocupacional é essencial para a saúde. ${ }^{15}$

"Déficit, doença ou lesão que afete qualquer componente de desempenho pode levar a uma falha na integração dos subsistemas componentes de desempenho, e, assim, resultar em falha ou distúrbio nas áreas de desempenho". ${ }^{15} \mathrm{~A}$ inabilidade para engajar ocupações e a incapacidade de realizar ati- 
vidades diárias comuns que o indivíduo quer ou necessita fazer, define a "disfunção ocupacional". ${ }^{17}$

Dessa maneira, a doença de Alzheimer leva a déficits nos componentes de desempenho ocupacional, o que por sua vez leva à deterioração em áreas de desempenho ocupacional, nos contextos do desempenho ocupacional em que são realizadas. ${ }^{18} \mathrm{Con}$ siderando que estes estejam afetados, o indivíduo com doença de Alzheimer apresenta uma disfunção ocupacional, justificando a intervenção do terapeuta ocupacional.

Essa intervenção tem sido extremamente diversificada, quanto às técnicas e teorias adotadas, em função das finalidades dos programas e serviços que a requisitam. As diversificações de posturas e ações significam, na verdade, as possibilidades de adequações de seus instrumentais em relação às finalidades dos programas e serviços, e das políticas sociais que os engendram e que, privilegiando determinados conceitos de Homem, Saúde e Doença, imprimem determinados modelos tecnológicos na assistência. ${ }^{12}$

A partir dessa caracterização, cabe apresentar as perspectivas filosóficas que dão origem às diferentes técnicas e teorias adotadas para a intervenção do terapeuta ocupacional, para melhor compreensão da abordagem proposta neste trabalho.

Existem duas diferentes perspectivas filosóficas: a visão reducionista e a visão holística. ${ }^{17,19}$ Essas perspectivas filosóficas fundamentam as várias teorias concernentes à natureza dos seres humanos e seus ambientes, inclusive as diversas teorias em terapia ocupacional.

A visão bolistica defende que o mundo percebido é indivisível, considerando que o todo é maior que a soma das partes, onde os elementos (abstratos e concretos) não podem ser compreendidos isoladamente. Considera a experiência individual, em sua relação com outros e com o ambiente.

A visão reducionista defende a busca da compreensão da natureza a partir das partes, sob uma visão objetiva e utilitária da realidade concreta. Estudando os componentes, as funções e as estruturas das partes, o todo pode ser compreendido.

Essas visões de mundo são denominadas como paradigmas da terapia ocupacional, pois todos os outros modelos ou estruturas de referência utilizados pelos terapeutas ocupacionais podem ser vistos encaixados nestas visões. ${ }^{17,19}$ Este artigo embasa-se na visão reducionista.

Apresentam-se, assim, os Modelos de Processo de Terapia Ocupacional Humanista, Positivista e Materialista Histórico. ${ }^{20} \mathrm{O}$ Modelo de Processo Humanista desenvolve-se na inexistência de padrões preestabelecidos, sendo o cliente que determina os caminhos a percorrer no processo terapêutico ocupacional, o que torna a relação terapêutica o centro deste processo; o Positivista visualiza o homem e a sociedade do ponto de vista da doença, o que caracteriza um processo terapêutico claro e definido, 
com uma estrutura rígida de procedimentos; e o Modelo de Processo Materialista Histórico é por definição criativo, transformador, questionador do contexto em que se efetiva, possibilitando a melhoria da qualidade de vida dos indivíduos "pela construção de um saber-fazer inserido nas práticas, nas relações e nas experiências do cotidiano". ${ }^{12}$

Desses modelos, é proposta a utilização do Modelo de Processo Positivista, pois este tem como foco principal tratar a doença, seguindo, para tanto, uma estrutura rígida de procedimentos. ${ }^{12} \mathrm{Tal}$ estrutura se configura num encadeamento de etapas distintas e logicamente ordenadas: encaminhamento, entrevista inicial, avaliação, planejamento de programa de tratamento, tratamento propriamente dito, reavaliações e alta. ${ }^{12}$

A estrutura rígida proposta pelo Modelo de Processo Positivista pode ser vista como possível para a intervenção com indivíduos com doença de Alzheimer em estágio inicial, pois focaliza o processo terapêutico a partir dos sintomas apresentados pelo indivíduo, de acordo com as etapas descritas. No tratamento dos indivíduos com doença de Alzheimer, estas etapas são seguidas conforme o modelo propõe. Vista a característica progressiva da doença, as etapas de reavaliação, de planejamento do programa de tratamento e de tratamento propriamente dito são realizadas a cada sinal de evolução ou involução do indivíduo, pois os objetivos da intervenção alteram de acordo com o quadro apresentado pelo indivíduo.
Partindo do Modelo de Processo Positivista, diversas estruturas de intervenção podem ser vistas como encaixadas e derivadas deste, seguindo a filosofia reducionista.

As estruturas de referência "formam um grupo de conceitos, definições, postulados e princípios internamente consistentes e inter-relacionados que propicia uma descrição sistemática e a prescrição para uma interação do profissional com seu domínio de interesse. Isto [...] serve como guia para suas ações em relação aos objetos humanos e nãohumanos". ${ }^{21}$ Assim, as estruturas de referência são organizadas, em primárias e em apli$\operatorname{cadas}^{17}$ (à prática terapêutica ocupacional).

As estruturas primárias de referência (EPR) utilizam-se das ciências básicas, exemplificando-se a fisiologia, a psicologia e a educação, como conhecimento emprestado para fundamentar as teorias que se aplicam à prática em terapia ocupacional. Dessa forma, a ciência básica psicológica deu origem à EPR psicológica. A elucidação desta se faz necessária para compreender a abordagem proposta neste artigo. ${ }^{17}$

A EPR psicológica baseia-se nos estudos científicos do comportamento e dos processos mentais, associados à neurofisiologia. A partir disso, tenta compreender o comportamento, o desenvolvimento humano, os aspectos psicológicos da aprendizagem e a cognição. Além disso, tenta entender o desenvolvimento da identidade pessoal e personalidade, papeis humanos e relações, e o comportamento das pessoas em grupos. ${ }^{17}$ 
Considerando esses aspectos, a EPR psicológica embasou o surgimento de várias estruturas aplicadas à prática terapêutica ocupacional, dentre as quais está a cognitiva, que pode ser utilizada para a intervenção com indivíduos em estágio inicial da doença de Alzheimer.

Conforme abordado anteriormente, tem-se que a doença de Alzheimer, por seu processo degenerativo, altera os componentes de desempenho, atingindo principalmente a integração cognitiva e os componentes cognitivos. Para intervir na integração cognitiva e nos componentes cognitivos, a estrutura aplicada de referência cognitiva se faz adequada, pois aborda as capacidades de informação do cérebro, sendo empregada majoritariamente quando se considera a disfunção cognitiva adquirida, sendo uma delas as condições cerebrais degenerativas, como as demências. ${ }^{17}$ Neste aspecto, o indivíduo com doença de Alzheimer poderá se beneficiar desta estrutura de intervenção, favorecendo sua integração cognitiva e seus componentes cognitivos e, consequentemente, suas áreas de desempenho nos contextos de desempenho que o indivíduo vivencia.

No entanto, é importante ressaltar a existência e validade de outras intervenções multidisciplinares, como o uso da medicação, a reabilitação neuropsicológica $(\mathrm{RN})$, que inclui a intervenção cognitiva, e o grupo informativo, com os pacientes, os familiares e os cuidadores. Tanto o uso da medicação como a RN têm apresentado resulta- dos positivos, como a melhora das funções cognitivas e do desempenho na realização das atividades de vida diária (AVD). ${ }^{8} \mathrm{~A}$ intervenção cognitiva pelo terapeuta ocupacional faz parte do trabalho multidisciplinar com o indivíduo com doença de Alzheimer.

Baseada nos fundamentos apresentados, a revisão de literatura mostra possível a intervenção cognitiva do terapeuta ocupacional com indivíduos com doença de Alzheimer, a qual será descrita a seguir.

\section{INTERVENÇÃO COGNITIVA DO TERAPEUTA OCUPACIONAL COM INDIVIDUUOS COM DOENÇA DE ALZHEIMER}

A doença de Alzheimer é progressiva e de um prognóstico difícil, com expectativas de melhoras limitadas. No entanto, as intervenções cognitivas podem organizar as atividades do paciente, promovendo uma melhor qualidade de vida. ${ }^{22}$

A base para a intervenção cognitiva é a crença na capacidade de regeneração do cérebro, conhecida como plasticidade cerebral. Caracterizada como uma mudança adaptativa na estrutura e funções do sistema nervoso, a plasticidade é um suporte orgânico à recuperação cognitiva, melhorando o déficit causado pela patologia. ${ }^{20}$ Neste contexto, acrescenta-se que a intervenção cognitiva serve como caminho para lentificar o processo degenerativo no estágio inicial, sobretudo em interação com estratégias medicamentosas. ${ }^{20}$ 
Esta intervenção cognitiva pode ser realizada de forma individualizada ou em grupo. As duas formas complementam-se. Enquanto a terapia individual permite direcionar o tratamento conforme a necessidade do paciente, a terapia em grupo proporciona um contato entre os pacientes, o que favorece habilidades comunicativas e a quebra da monotonia. Dessa forma, o uso combinado de intervenções individual e grupal é recomendado para obter sucesso mais amplo no tratamento. ${ }^{20}$

Independentemente da forma de intervenção, o paciente deverá passar pelos processos de avaliação, terapia propriamente dita e alta, equivalendo-se com o modelo de processo positivista apresentado anteriormente. $O$ processo de avaliação é de significativa importância, pois permite ao terapeuta ocupacional ter ciência de quais componentes estão deficitários e quais estão preservados, possibilitando decidir em que direção o tratamento será realizado. ${ }^{20}$

Os processos de avaliação permitem ainda monitorar a progressão da doença de Alzheimer e avaliar o nível de benefício das intervenções. ${ }^{23}$ A partir disso, o terapeuta ocupacional poderá intervir para abordar a relação entre processos falhos, preservados e estratégias compensatórias. Essas intervenções são possíveis em pacientes com prejuízos cognitivos de leves a moderados, pois estes ainda possuem a capacidade de aprendizagem e podem beneficiar-se de estratégias para compensar a perda das habilidades. ${ }^{20}$
Dessa forma, e lembrando que a ocupação humana é o foco da intervenção do terapeuta ocupacional, ressalta-se a importância de manter preservada pelo maior tempo possível a integridade da ocupação, através da realização de atividades, pois: ${ }^{23}$

- o fazer, a ação, o agir, são necessidades de todo ser humano;

- reverter o processo da doença ainda não é possível, mas o fato de o paciente realizar atividades estimula-o a usar suas capacidades remanescentes e o ajuda a mantê-las; é um trabalho de prevenção e manutenção;

- a atividade é um meio para a comunicação verbal e não-verbal;

- a realização de atividades terapêuticas ocupacionais favorece a qualidade de vida, uma vez que permite sua realização dentro dos limites de autonomia e independência do indivíduo;

- a atividade dá um sentido de identidade, de prazer, ajuda a preservar a dignidade da pessoa.

Para que a realização de atividades cumpra seu papel terapêutico com os indivíduos com doença de Alzheimer, as atividades devem ser simplificadas, fragmentadas em pequenas tarefas, repetitivas, rotineiras, familiares e adaptadas na medida em que as habilidades do cliente vão diminuindo. ${ }^{23}$ Ressalta-se, ainda, a importância de o terapeuta ocupacional perceber e correlacionar a perda cognitiva com o declínio das habili- 
dades funcionais, abordando a realização das atividades de vida diária, atividades de trabalho e produtivas, e atividades de lazer ou de diversão. ${ }^{23}$

A evolução da doença de Alzheimer pode ser retardada e estratégias de intervenção cognitiva podem melhorar a qualidade de vida, a autonomia funcional e o estado afetivo dos pacientes e seus familiares. ${ }^{20}$ Portanto, define-se que o papel do profissional que atua com indivíduos com doença de Alzheimer está em melhorar a qualidade de vida e o bem-estar deste, acreditando que se pode reconstruir o que estiver alterado, compensar o que foi perdido, utilizando o que estiver conservado. ${ }^{24}$

Dessa forma, cabe ao terapeuta ocupacional empenhar-se na prevenção do agravamento progressivo dos principais sintomas cognitivos, visando à otimização da qualidade de vida do indivíduo com doença de Alzheimer, contando, para isto, além da contribuição de outros profissionais, com a participação direta no tratamento tanto do próprio paciente, como da família e/ou do cuidador e da comunidade.

Assim, apesar de o processo terapêutico apresentado neste trabalho se basear no modelo de processo positivista, o qual enfoca o paciente e sua doença, o envolvimento familiar/social no tratamento e a busca por um ambiente acolhedor fazem com que o terapeuta ocupacional aborde também estes aspectos, utilizando a visão humanista como complementar. Dessa forma, o processo terapêutico estará centrado não so- mente na doença, mas também na relação estabelecida entre o indivíduo com doença de Alzheimer, sua rede de suporte e o terapeuta ocupacional.

\section{CONCLUSÃO}

A partir da revisão bibliográfica realizada, foi possível correlacionar a teoria e a prática da intervenção terapêutica ocupacional junto aos indivíduos com doença de Alzheimer, com um enfoque sobre os aspectos cognitivos, pois estes aspectos que principalmente são gradativamente afetados nesta doença e levam ao comprometimento do desempenho ocupacional competente.

Por fim, ressalta-se a importância da intervenção terapêutica ocupacional com indivíduos com doença de Alzheimer na manutenção da integração cognitiva e dos componentes cognitivos. A alteração destes componentes de desempenho afeta as áreas de desempenho nos contextos de desempenho em que o indivíduo está inserido. A intervenção direta nesses componentes estimula a utilização dos mesmos, favorecendo o desempenho ocupacional competente e, portanto, a independência do indivíduo com doença de Alzheimer.

Deve-se considerar que, como a doença de Alzheimer é progressiva, a intervenção cognitiva não deterá o avanço da doença, apenas fará com que o indivíduo mantenha seu nível mais elevado de habilidades e funcionamento por um maior período de tempo. 
Ao longo deste trabalho, a revisão de literatura mostrou válida a utilização da visão reducionista aplicando-se o modelo de processo positivista, visto ser esta estruturada para a intervenção na integração cognitiva e nos componentes cognitivos. No entanto, o envolvimento da rede de suporte do indivíduo com doença de
Alzheimer é inerente ao processo terapêutico ocupacional. Dessa forma, percebese a possibilidade e a necessidade de utilização do Modelo de Processo Humanista para a mesma clientela, o que possibilitará a visão holística das experiências individuais em relação com os outros e com o ambiente.

\section{REFERÊNCIAS}

1 Papalia DE, Olds SW. Terceira idade e o fim da vida. In: Papalia DE, Olds SW. Desenvolvimento Humano. Porto Alegre: Artes Médicas Sul; 2000. p. 489-554.

2 Trentini CM, Xavier FMF, Fleck MPA. Qualidade de vida em idosos. In: Parente MAMP. Cognição e envelhecimento. Porto Alegre: Artmed; 2006. p. 19-29.

3 Organização Mundial de Saúde. Envelhecimento ativo: uma política de saúde. Brasília: Organização PanAmericana de Saúde; 2005.

4 Motta MP, Ferrari MAC. Intervenção terapêutico-ocupacional junto a indivíduos com comprometimento no processo de envelhecimento. In: De Carlo MMRP; Luzo MCM, orgs. Terapia Ocupacional: reabilitação física e contextos hospitalares. São Paulo: Roca; 2004. p.293-303.

5 Zimerman GI. Velhice: aspectos biopsicossociais. Porto Alegre: Artmed; 2005.

6 Lei 10.741 Estatuto do Idoso 2003 out 1. Pub DO 1(1), [2003 out 1].

7 Avila R, Miotto EC. Funções executivas no envelhecimento normal e na doença de Alzheimer. J Bras Psiquiatr 2003 jan-fev [acesso em: 2006 jun 11]; p.53-63.

Disponível em: URL: < http:// www.bireme.org $>$.

8 Brasil. Ministério da Saúde. Mal de Alzheimer atinge 6\% dos idosos brasileiros. Disponível em: URL: < http:/ /portal.saude.gov.br/ > .

9 Associação Brasileira de Alzheimer. Relatório informa que demência vai quadruplicar. [acesso em: 2006 set 24]Disponível em: URL: < http:// www.abraz.org.br $>$.

10 Grieve J. Neuropsicologia em terapia ocupacional: exame da percepção e cognição. São Paulo: Santos; 2005.

11 Parente MAMP, Wagner GP. Teorias abrangentes sobre envelhecimento Cognitivo. In: Parente MAMP, editor, et al. Cognição e envelhecimento. Porto Alegre: Artmed; 2006. Cap. 2.

12 Medeiros MHR. Terapia Ocupacional: um enfoque epistemológico e social. São Carlos: EdUFSCAR; 2003.

13 Francisco BR. Terapia ocupacional. 2. ed. Campinas: Papirus; 2001.

14 Neistadt ME, Crepeau EB. Introdução à terapia ocupacional. In: Neistadt ME, Crepeau EB, orgs. Terapia ocupacional Willard \& Spackman. 9. ed. Rio de Janeiro: Guanabara Koogan; 2002. p.3-9. 
15 Pedretti LW, Early MB. Desempenho ocupacional e modelos de prática para disfunção física. In: Pedretti LW, Early MB, orgs. Terapia ocupacional: capacidades práticas para as disfunções físicas. São Paulo: Roca; 2005. Cap.1.

16 American Occupational Therapy Association - AOTA. Terminologia uniforme para a terapia ocupacional. In: Neistadt ME, Crepeau EB, orgs. Terapia ocupacional - Willard \& Spackman. 9. ed. Rio de Janeiro: Guanabara Koogan; 2002. Apêndice F.

17 Hagedorn R. Fundamentos para a prática em terapia ocupacional. São Paulo: Roca; 2003.

18 Glogoski C. Doença de Alzheimer. In: Pedretti LW, Early MB, orgs. Terapia ocupacional: capacidades práticas para as disfunções físicas. São Paulo: Roca; 2005. p.742-51.
19 Hagedorn R. Fundamentos para a prática em terapia ocupacional. São Paulo: Roca; 1999.

20 Holderbaum CS, et al. A intervenção cognitiva para pacientes portadores de demência do tipo Alzheimer. In: Parente MAMP, et al. Cognição e envelhecimento. Porto Alegre: Artmed; 2006. Cap.16.

21 Reed KL. Teoria e estrutura de referência. In: Neistadt ME, Crepeau EB, orgs. Terapia Ocupacional - Willard \& Spackman. 9. ed. Rio de Janeiro: Guanabara Koogan; 2002. p. 485-7.

22 Parente MAMP, et al. Cognição e envelhecimento. Porto Alegre: Artmed; 2006.

23 Ferrari MACF. A terapia ocupacional na reabilitação do portador de Alzheimer. Mundo da Saúde 2001 out-dez; 25(4): p. 425-29.

24 Camara VD, et al. Atendimento interdisciplinar a pacientes com demência e seus cuidadores. Arquivos de Geriatria e Gerontologia 1998; 2(1): p.11-21. 